\section{This paper is published as part of a $P C C P$ themed issue on recent developments in X-ray absorption spectroscopy}

\section{Guest Editor: Jeroen Anton van Bokhoven}

Editorial

Recent developments in X-ray absorption spectroscopy J. A. van Bokhoven, Phys. Chem. Chem. Phys., 2010 DOI: $10.1039 / \mathrm{c0cp90010a}$

Perspectives

Parameter-free calculations of X-ray spectra with FEFF9 John J. Rehr, Joshua J. Kas, Fernando D. Vila, Micah P. Prange and Kevin Jorissen, Phys. Chem. Chem. Phys., 2010

DOI: $\underline{10.1039 / \mathrm{b} 926434 \mathrm{e}}$

The atomic AXAFS and $\Delta \mu$ XANES techniques as applied to heterogeneous catalysis and electrocatalysis D. E. Ramaker and D. C. Koningsberger, Phys. Chem. Chem. Phys., 2010

DOI: $\underline{10.1039 / \mathrm{b} 927120 \mathrm{c}}$

Advances in high brilliance energy dispersive X-ray absorption spectroscopy

Sakura Pascarelli and Olivier Mathon, Phys. Chem. Chem. Phys., 2010

DOI: $10.1039 / \mathrm{b} 926509 \mathrm{k}$

Communication

$\mu$-XANES mapping of buried interfaces: pushing microbeam techniques to the nanoscale

Paolo Ghigna, Sonia Pin, Giorgio Spinolo, Mark A. Newton, Michele Zema, Serena C. Tarantino, Giancarlo Capitani and Francesco Tatti, Phys. Chem. Chem. Phys., 2010 DOI: $10.1039 / \mathrm{c} 000195 \mathrm{C}$

Papers

L-edge XANES analysis of photoexcited metal complexes in solution

Renske M. van der Veen, Joshua J. Kas, Christopher J. Milne, Van-Thai Pham, Amal El Nahhas, Frederico A. Lima, Dimali A. Vithanage, John J. Rehr, Rafael Abela and Majed Chergui, Phys. Chem. Chem. Phys., 2010

DOI: $10.1039 / \mathrm{b} 927033 \mathrm{~g}$

EXAFS as a tool to interrogate the size and shape of mono and bimetallic catalyst nanoparticles

Andrew M. Beale and Bert M. Weckhuysen, Phys. Chem.

Chem. Phys., 2010

DOI: $\underline{10.1039 / \mathrm{b} 925206 \mathrm{a}}$

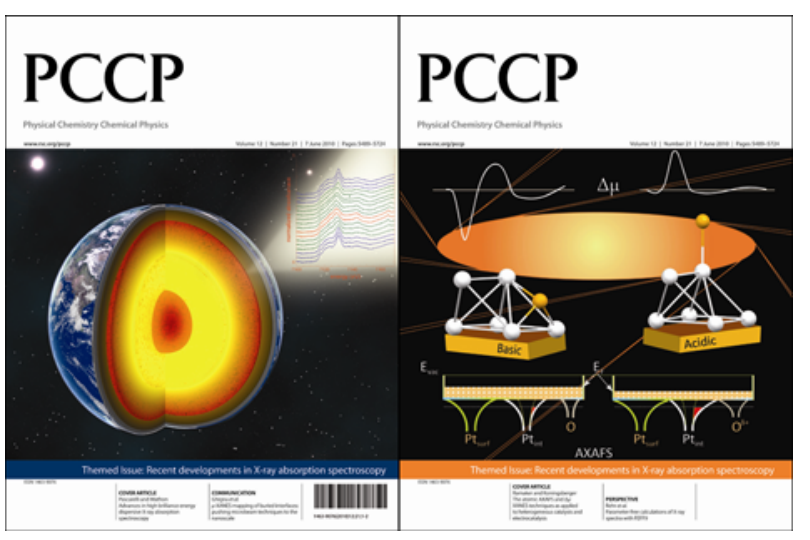

X-Ray absorption in homogeneous catalysis research: the iron-catalyzed Michael addition reaction by XAS, RIXS and multi-dimensional spectroscopy Matthias Bauer and Christoph Gastl, Phys. Chem. Chem. Phys., 2010

DOI: $\underline{10.1039 / b 926385 c}$

Combined TPRx, in situ GISAXS and GIXAS studies of model semiconductor-supported platinum catalysts in the hydrogenation of ethene

Sonja A. Wyrzgol, Susanne Schäfer, Sungsik Lee,

Byeongdu Lee, Marcel Di Vece, Xuebing Li, Sönke Seifert, Randall E. Winans, Martin Stutzmann, Johannes A. Lercher and Stefan Vajda, Phys. Chem. Chem. Phys., 2010 DOI: $\underline{10.1039 / \mathrm{b} 926493 \mathrm{k}}$

Near sulfur L-edge X-ray absorption spectra of methanethiol in isolation and adsorbed on a Au(111) surface: a theoretical study using the four-component static exchange approximation

Sebastien Villaume, Ulf Ekström, Henrik Ottosson and Patrick Norman, Phys. Chem. Chem. Phys., 2010 DOI: $\underline{10.1039 / b 926109 e}$

Influence of additives in defining the active phase of the ethylene oxychlorination catalyst

N. B. Muddada, U. Olsbye, L. Caccialupi, F. Cavani, G. Leofanti, D. Gianolio, S. Bordiga and C. Lamberti, Phys. Chem. Chem. Phys., 2010

DOI: $10.1039 / \mathrm{b} 926502 \mathrm{n}$

First-principles calculations of X-ray absorption spectra at the K-edge of $3 d$ transition metals: an electronic structure analysis of the pre-edge Delphine Cabaret, Amélie Bordage, Amélie Juhin, Mounir Arfaoui and Emilie Gaudry, Phys. Chem. Chem. Phys., 2010

DOI: $10.1039 / \mathrm{b} 926499 \mathrm{j}$

First steps in combining modulation excitation spectroscopy with synchronous dispersive EXAFS/DRIFTS/mass spectrometry for in situ time resolved study of heterogeneous catalysts Davide Ferri, M. Santosh Kumar, Ronny Wirz, Arnim Eyssler, Oxana Korsak, Paul Hug, Anke Weidenkaff and Mark A. Newton, Phys. Chem. Chem. Phys., 2010 DOI: $\underline{10.1039 / \mathrm{b} 926886 \mathrm{c}}$

Novel opportunities for time-resolved absorption spectroscopy at the X-ray free electron laser B. D. Patterson and R. Abela, Phys. Chem. Chem. Phys., 2010

DOI: $10.1039 / \mathrm{c003406a}$ 
Spatially resolved 3D micro-XANES by a confocal detection scheme

Geert Silversmit, Bart Vekemans, Sergey Nikitenko, Sylvia

Schmitz, Tom Schoonjans, Frank E. Brenker and Laszlo

Vincze, Phys. Chem. Chem. Phys., 2010

DOI: $\underline{10.1039 / \mathrm{c004103n}}$

Wavelet transform EXAFS analysis of mono- and dimolybdate model compounds and a Mo/HZSM-5

dehydroaromatization catalyst

Robert O. Savinelli and Susannah L. Scott, Phys. Chem.

Chem. Phys., 2010

DOI: $10.1039 / \mathrm{b} 926474 \mathrm{~d}$

Electronic structure of alumina-supported monometallic Pt and bimetallic PtSn catalysts under hydrogen and carbon monoxide environment

Jagdeep Singh, Ryan C. Nelson, Brian C. Vicente,

Susannah L. Scott and Jeroen A. van Bokhoven, Phys.

Chem. Chem. Phys., 2010

DOI: $\underline{10.1039 / \mathrm{c} 000403 \mathrm{k}}$

\section{Determination of $\mathrm{CO}, \mathrm{H}_{2} \mathrm{O}$ and $\mathrm{H}_{2}$ coverage by XANES} and EXAFS on Pt and Au during water gas shift reaction Neng Guo, Bradley R. Fingland, W. Damion Williams, Vincent F. Kispersky, Jelena Jelic, W. Nicholas Delgass, Fabio H. Ribeiro, Randall J. Meyer and Jeffrey T. Miller, Phys. Chem. Chem. Phys., 2010

DOI: $\underline{10.1039 / \mathrm{c000240m}}$

Complementarity between high-energy photoelectron and L-edge spectroscopy for probing the electronic structure of $5 \mathrm{~d}$ transition metal catalysts

Toyli Anniyev, Hirohito Ogasawara, Mathias P. Ljungberg, Kjartan T. Wikfeldt, Janay B. MacNaughton, Lars-Åke Näslund, Uwe Bergmann, Shirlaine Koh, Peter Strasser, Lars G.M. Pettersson and Anders Nilsson, Phys. Chem.

Chem. Phys., 2010

DOI: $10.1039 / \mathrm{b} 926414 \mathrm{k}$

In situ time-resolved DXAFS for the determination of kinetics of structural changes of H-ZSM-5-supported active Re-cluster catalyst in the direct phenol synthesis from benzene and $\mathrm{O}_{2}$

Mizuki Tada, Yohei Uemura, Rajaram Bal, Yasuhiro Inada, Masaharu Nomura and Yasuhiro Iwasawa, Phys. Chem.

Chem. Phys., 2010

DOI: $10.1039 / \mathrm{c000843p}$

Sulfur poisoning mechanism of steam reforming catalysts: an X-ray absorption near edge structure (XANES) spectroscopic study

Yongsheng Chen, Chao Xie, Yan Li, Chunshan Song and

Trudy B. Bolin, Phys. Chem. Chem. Phys., 2010

DOI: $\underline{10.1039 / \mathrm{b} 925910 \mathrm{~b}}$

Peroxide-like intermediate observed at hydrogen rich condition on $\mathrm{Pt}(111)$ after interaction with oxygen

Janay B. MacNaughton, Lars-Åke Näslund, Toyli Anniyev, Hirohito Ogasawara and Anders Nilsson, Phys. Chem.

Chem. Phys., 2010

DOI: 10.1039/b926409b 


\title{
EXAFS as a tool to interrogate the size and shape of mono and bimetallic catalyst nanoparticles $\dagger$
}

\author{
Andrew M. Beale* and Bert M. Weckhuysen \\ Received 30th November 2009, Accepted 9th March 2010 \\ First published as an Advance Article on the web 8th April 2010 \\ DOI: $10.1039 / \mathbf{b 9 2 5 2 0 6 a}$
}

The influence of atom packing and the geometric arrangement of atoms on the coordination number(s) and resultant simulated EXAFS spectra for face centrered cubic (fcc), hexagonal close packed (hcp) and body centered cubic (bcc) monometallic structures and fcc bimetallic structures, has been examined for metal clusters with an isotropic diameter up to $3 \mathrm{~nm}$. We observe clear differences as a function of size and shape for all types of packing; in particular the surface aspect ratios (surface-to-bulk) of the shapes strongly influences the 'growth curves' as a function of the number of atoms for all packing types examined. Discrimination between the different types of structure based on the coordination shell occupancy appears possible, although is dependent on the type of packing. For fcc and hcp packing, structures comprising less than 200 atoms, and particularly less than 100 atoms, exhibit strong variation in the first shell coordination number as a function of shape. For bcc structures this dependency of the coordination shell number for small clusters is much less pronounced and in some cases (for higher shell ( $>$ N4) coordination numbers) an opposite trend is observed in that the difference in coordination number as a function of shape becomes more marked with an increasing number of atoms. For the fcc bimetallic systems, model structures possessing distinct bimetal distributions, including both non-random and random alloy types, were simulated and examined. Clear variations in both the 1 st shell coordination number and in the simulated spectra were observed as a function of the bimetal structure. A dual edge analysis of the data and subsequent examination of the coordination shell numbers as a function of the bimetal distribution enables for the size, composition and distribution of the each species, to be determined.

\section{Introduction}

Metal nanoparticles have unique chemical and physical properties that make them attractive for use in a number of research areas. In particular they comprise the active component of a number of supported metal catalysts. ${ }^{1,2}$ Crucial to the understanding of the activity that they possess, is a thorough understanding of the role(s) that their size, shape and extent of interaction with the support play. However, due to their small nature, they are often difficult to characterize. Microscopic techniques such as transmission electron microscopy (TEM) represent the most appropriate techniques to do so, although it is difficult to obtain a true representation of the sample and the samples can often be difficult to measure due to their instability in the electron beam. Other techniques that can yield such information include X-ray diffraction (XRD) and chemisorption although the former is essentially limited to studying particles $>3 \mathrm{~nm}$ (although this can be smaller when using high energy diffraction), ${ }^{3}$ whilst the latter is limited in its application. An alternative technique which has often been used to

Inorganic Chemistry and Catalysis, Debye Institute for

NanoMaterials Science, Utrecht University, Sorbonnelaan 16,

3584 CA, Utrecht, the Netherlands.E-mail: A.M.Beale@uu.nl;

Fax: 0031 (0) 30251 1027; Tel: 0031 (0) 302536815

$\dagger$ Electronic supplementary information (ESI) available: Hill plots; simulated EXAFS data. See DOI: 10.1039/b925206a characterize these samples is X-ray absorption spectroscopy (XAFS) and in particular from the extended region (EXAFS); a bulk sampling technique which can also be employed to study samples under in situ conditions. ${ }^{4-6}$ Although it is well documented that a good estimation of particle size can be gleaned from a first shell analysis (often it is assumed that the particle is spherical), information regarding the shape of the particle really needs to be obtained from an analysis/fitting of the higher shells of the EXAFS data. To date there have been a number of attempts to do this and in particular, to propose models to describe the influence of shape and crystal packing on structural coordination numbers. These works include that of Greegor and Lytle, published in the 1980s, in which they described hypothetical models for the spherical, cubic and disc shaped active components of supported face centred cubic (fcc) metal catalysts; ${ }^{7}$ Benfield in which the influence of packing/cluster geometry (cuboctahedron and icosahedron) on the N1 value (first coordination sphere) was examined; ${ }^{8}$ Montejano-Carrizales et al. whom demonstrated through a detailed systematic enumeration of the geometrical characteristics of cuboctohedral, icosahedral, body-centred and primitive spherical structures how cluster packing influenced coordination numbers at both surfaces and in the bulk $;{ }^{9}$ Jentys who determined the influence of a number of particle shapes (spheres, cubes and distorted cubes), up to the 5th coordination sphere, on the coordination number and utilised hyperbolic functions to fit 
this data to extrapolate the findings in order to allow for the determine of the shape of bigger particle sizes ${ }^{10}$ and finally Frenkel whom described both the influence of crystal packing (including for the first time hexagonal close packed structures (hcp)) and 2nd metal (bimetal structures) loading on the coordination numbers. ${ }^{10,11}$ Although these excellent efforts have no doubt afforded researchers the opportunity to obtain more from the EXAFS data than is normally possible, they are somewhat limited in their scope of application. This is for two reasons. Firstly, the number of different shapes that are described is limited essentially to 6 (spheres, discs, cubes, slabs, distorted cubes, icosahedra). With the advances made in the understanding of catalyst preparation, it has been possible for researchers to prepare/control the shapes of the active component $e . g$. the formation of cylindrical-type nano-gold species in the channels of SBA-15, polyvinyl pyrrolidone (PVP) capped cubic Pt nano-cubes and pyramidal gold structures on gold $\left(\begin{array}{lll}1 & 0 & 0\end{array}\right)$ surfaces to illustrate but a few. ${ }^{12-17}$ A number of studies now exist in which a clear influence of particle shape and size on activity has been shown. ${ }^{13,18,19}$ Thus there is a need to expand upon the number of structures for which such coordination number influence has been considered. Secondly and perhaps more importantly, until recently there has been no such study performed on hexagonal close packed (hcp) or body-centered cubic (bcc) systems. ${ }^{20}$ Essentially this is due to the fact that the majority of active catalysts (e.g. $\mathrm{Pt}, \mathrm{Au}, \mathrm{Ag}, \mathrm{Rh}, \mathrm{Pd}, \mathrm{Ni}, \mathrm{Cu}$ etc.) in the bulk phase, possess an fcc structure. However this accounts for less than $1 / 3$ of the packing of metallic structures in their elemental form at room temperature (we estimate that up to element $82(\mathrm{~Pb})$, the distribution of the different packing arrangements correspond to fcc $(28 \%)$, bcc $(29 \%)$ and hcp (43\%) respectively). Due to the recent increasing interest in catalytic materials possessing hep and bcc structures (in particular for example, cobalt and iron containing Fischer-Tropsch synthesis catalysts), ${ }^{21-29}$ there is clearly a need to provide similar information regarding the influence of size and shape on shell coordination numbers for these packing systems as well.

In this article we determine and examine the influence of particle size, the shape and the packing on the coordination number for a series of nano-sized metal cluster shapes comprising spheres (including, cuboctahedron, icosahedron and decahedron), cubes, square-based pyramids, cylinders and half-spheres for fcc, hcp and bcc systems up to a maximum isotropic diameter of $3 \mathrm{~nm}$. In addition, we demonstrate, using a similar approach to that used previously, how the ratio between the derived coordination numbers varies as a function of shape and how such information could be utilised/obtained from real EXAFS data. ${ }^{10} \mathrm{We}$ simulate also how these differences manifest themselves in actual EXAFS spectra. Finally we conclude with an examination of the influence of bimetal distribution and loading on the coordination numbers for a series of contrived spherical structures of $c a .1 .3 \mathrm{~nm}$ diameter and how EXAFS can be used to interrogate the structure to obtain this information, something which, until recently had hardly been examined in the literature despite the importance of such metallic species for catalysis. ${ }^{11,30}$

\section{Experimental}

The hypothetical metal clusters were based on the bulk structures of $\mathrm{Pt}, \mathrm{Co}$ and $\mathrm{Fe}$ which consist of a fcc/hcp/bcc lattices with a unit cell size of $3.96 \AA$ (Pt), 3.05 by $4.06 \AA$ (Co) and $2.82 \AA(\mathrm{Fe})$ respectively. ${ }^{31-33}$ Magic number clusters $(13,55,147,309$ and 561) were created for Pt structures from a database of spherical cluster geometric coordinates where the point coordinates were adjusted so that the closest $\mathrm{Pt}-\mathrm{Pt}$ distances were fixed to that of bulk Pt $(2.75 \AA) .{ }^{34}$ Table 1 contains a list of the shell parameters used for $\mathrm{Pt} / \mathrm{Co} / \mathrm{Fe}$ respectively. The shell distances and coordination numbers were obtained from the respective crystal reference structures and after a least squares refinement of the reference foil. Clusters consisting of hcp Co, bcc Fe, non-spherical Pt clusters and the bimetallic systems were created using the nanocluster building function of the Materials Studio ${ }^{\mathbb{R}}$ programme by a gradual increase of the maximum radial distance. The nonspherical shapes profiled were as follows: cube, square-based pyramid, half-sphere and cylinder; structures consisting of square-based pyramids and cylinders were built up by varying either base length and cluster height (pyramids) or the radius and height (cylinders) using the 001 plane as the base plane. Clusters were created containing up to a maximum of 561 atoms (this also happens to also correspond to a large fcc cuboctahedron $c a .3 \mathrm{~nm}$ in size). The average coordination of up to 5 shells for fcc Pt, 8 for hep Co and 7 for bcc Fe, as well as the surface-to-bulk ratio (determined as a ratio of fully coordinated : non-fully coordinated atoms in the cluster) were then determined using macros developed in-house; details of the reference structures plotted and used in this study are given in Table 2 with an illustration of some of the simulations (spherical particles containing 147 (fcc), 156 (hcp) and 137 (bcc) atoms, respectively) shown in the radial distribution function (RDF) histogram given in Fig. 1. Note that in order to directly compare only the influence of the packing of similar sized atoms on the radial distribution function, the pattern for the 147 atom containing fcc structure was adjusted to reflect that of $\mathrm{Fe}$ instead of $\mathrm{Pt}^{32} \mathrm{We}$ observe at this stage that the greater number of shells observed in the radial distribution function of the icosahedron and decahedron are due to stacking faults which are necessary to arrive at these particular structural arrangements. ${ }^{11,17}$

Given in Table 3 are the results from the simulation for the growth curves for the average first coordination number $v s$. number of atoms for the first shell (N1) for each of the packing types. The data were simulated by performing a non linear least-squares fitting using a Hill function (eqn (1)), with $V_{\text {Max }}$ set respectively to 12 (fcc, hcp) and 8 (bcc) representing a hypothetical bulk/maximum coordination respectively. Simulations were performed on both the idealized spherical models as well as on the total data (thereby generating an average function for all of the profiled shapes) respectively. The results from the data fitting are given in ESI, Fig. $1 . \dagger$

$$
y=V_{\operatorname{Max}} x^{n} /\left(k^{n}+x^{n}\right)
$$

The bimetallic structure models used in this study comprise a mixture of $\mathrm{Pt}$ and $\mathrm{Pd}$ and were confined to a 137 atom containing spherical cluster of $c a .1 .3 \mathrm{~nm}$ diameter. 8 model 
(a)

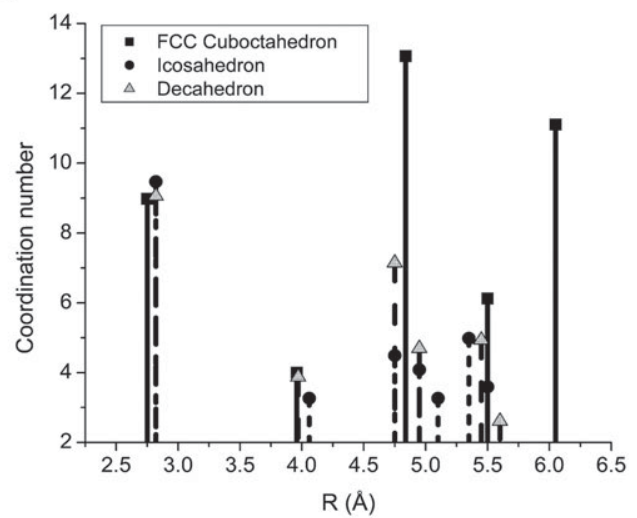

(b)

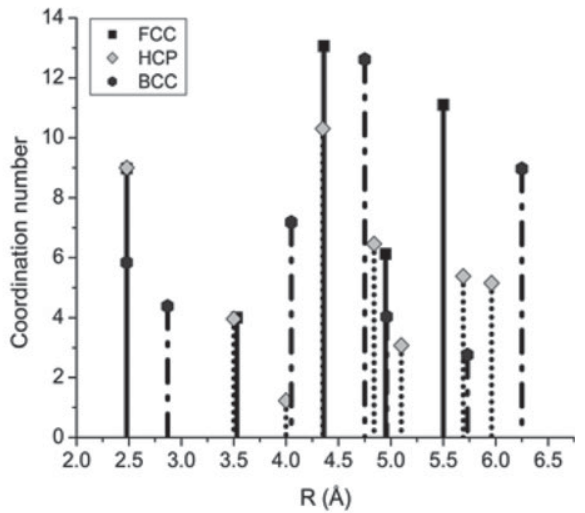

(c)

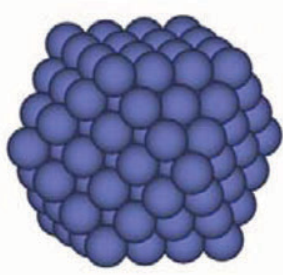

FCC Cuboctahedron (147)

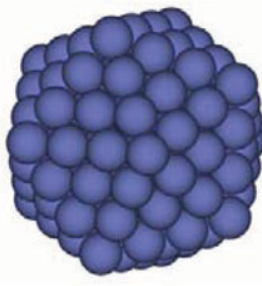

Icosahedron

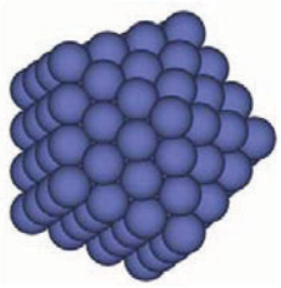

Decahedron
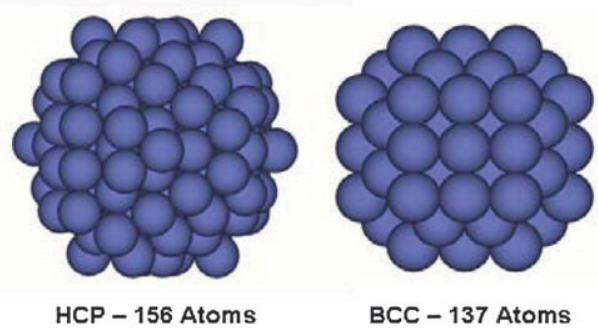

Fig. 1 Histograms detailing the influence of atom packing on the higher shell coordination for (a) 147 atom Pt-containing cuboctahedron, icosahedron, decahedron and (c) 147 atom (fcc), 156 atom (hcp) and 137 atom (bcc) clusters, respectively. Note that a binning window of $0.15 \AA$ was used to differentiate between shell radial distances for the icosahedron. In (b) are shown the corresponding histograms.

Table 1 Shell parameters for Pt (fcc), Co (hcp) and Fe (bcc) structures used for the nano-particle structure generation

\begin{tabular}{|c|c|c|c|c|c|c|c|c|}
\hline & Shell 1 & Shell 2 & Shell 3 & Shell 4 & Shell 5 & Shell 6 & Shell 7 & Shell 8 \\
\hline Pt-Pt (fcc) Shell distance & 2.75 & 3.96 & 4.84 & 5.50 & 6.05 & & & \\
\hline Coordination number & 12 & 6 & 24 & 12 & 24 & & & \\
\hline Co-Co (hcp) Shell distance & 2.50 & 3.5 & 4.0 & 4.35 & 4.84 & 5.10 & 5.69 & 5.96 \\
\hline Coordination number & 12 & 6 & 2 & 18 & 12 & 16 & 12 & 12 \\
\hline $\mathrm{Fe}-\mathrm{Fe}$ (bcc) Shell distance & 2.49 & 2.87 & 4.05 & 4.75 & 4.96 & 5.73 & 6.24 & \\
\hline Coordination number & 8 & 6 & 12 & 24 & 8 & 6 & 24 & \\
\hline
\end{tabular}

structures were examined labelled bi-cluster, single-half, core-shell, random, decorated, bi-cluster homo and bi-cluster inhomo accordingly. The last two structures were chosen particularly to simulate a non uniform distribution of bimetallic particles. Co hcp and Fe bcc clusters were not simulated since often hcp and bcc structures, when mixed with fcc metals tend to adopt the fcc structure. ${ }^{30,35}$

Spectral simulations on the influence of the coordination number on the EXAFS spectra were also performed. For every atom of the model clusters an individual EXAFS spectrum was created by generating an input file which could be read into the EXCURV98 programme, with the individual spectra then being summed to obtain an average signal. The resultant spectra were plotted over a $k$-range $3.4-17 \AA$ ( $R$-range of 1-6 $\AA$ ) and were $k^{3}$ weighted. The parameters used to generate the input files, e.g. E0, $\mathrm{S}^{2}$ and the Debye-Waller factor were obtained from the fitting of $10 \mu \mathrm{m} \mathrm{Pt} 3$ different foils from 2 different beamlines (BM26 A (DUBBLE) at the ESRF and Station 7.1 (Daresbury) at the SRS) were compared and the signals were found to vary by $<5 \%$ ) and $\mathrm{Pd}$ reference foils measured at the $\mathrm{L}_{\mathrm{III}}$ and K-edges (11.564 and $24.350 \mathrm{eV}$ ) at room temperature, respectively. ${ }^{36}$ Table 4 contains a list of 
Table 2 (a) Cluster shapes, dimensions and first coordination number for the fcc and hep clusters simulated in this study; (b) cluster packing, number of atoms (magic number based) and first coordination number for cuboctahedron, icosahedron and decahedron clusters

\begin{tabular}{|c|c|c|c|c|c|c|c|c|}
\hline \multicolumn{3}{|l|}{ (a) fcc } & \multicolumn{3}{|l|}{ hcp } & \multicolumn{3}{|l|}{ bcc } \\
\hline Shape/size/nm & No. atoms & First shell CN & Shape/size/nm & No. atoms & First shell CN & Shape/size/nm & No. atoms & First shell CN \\
\hline \multicolumn{9}{|l|}{ Sphere } \\
\hline 3 & 561 & 10.06 & 2.3 & 510 & 9.99 & 2.4 & 537 & 6.77 \\
\hline 2 & 309 & 9.63 & 2.1 & 382 & 9.75 & 2.0 & 307 & 6.41 \\
\hline 1.5 & 147 & 8.98 & 1.7 & 194 & 9.19 & 1.7 & 137 & 5.84 \\
\hline 0.6 & 55 & 7.85 & 1.3 & 86 & 8.35 & 1.2 & 59 & 5.42 \\
\hline \multirow[t]{2}{*}{0.3} & 13 & 5.53 & 1.1 & 48 & 7.75 & 0.7 & 15 & 4.27 \\
\hline & & & 0.7 & 10 & 5.3 & & & \\
\hline \multicolumn{9}{|l|}{ Cubic } \\
\hline $1.9 \times 1.9 \times 1.9$ & 500 & 9.75 & $1.9 \times 1.9 \times 1.9$ & 540 & 9.90 & $1.9 \times 1.9 \times 1.9$ & 550 & 6.27 \\
\hline $1.8 \times 1.8 \times 1.8$ & 365 & 9.47 & $1.6 \times 1.6 \times 1.6$ & 294 & 9.44 & $1.8 \times 1.8 \times 1.8$ & 432 & 6.19 \\
\hline $1.6 \times 1.6 \times 1.6$ & 256 & 9.19 & $1.3 \times 1.3 \times 1.3$ & 165 & 8.79 & $1.6 \times 1.6 \times 1.6$ & 341 & 5.87 \\
\hline $1.1 \times 1.1 \times 1.1$ & 108 & 8.33 & $0.9 \times 0.9 \times 0.9$ & 56 & 7.75 & $1.3 \times 1.3 \times 1.3$ & 189 & 5.42 \\
\hline \multirow{2}{*}{\multicolumn{9}{|c|}{ Pyramid $^{a} \times 0.0$}} \\
\hline & & & & & & & & \\
\hline $3.0 \times 3.0 \times 3.0$ & 550 & 9.30 & $2.7 \times 2.7 \times 2.7$ & 534 & 9.60 & $3.0 \times 3.0 \times 1.8$ & 506 & 6.09 \\
\hline $2.6 \times 2.6 \times 2.6$ & 398 & 9.10 & $2.5 \times 2.5 \times 2.5$ & 420 & 9.45 & $2.7 \times 2.7 \times 1.6$ & 385 & 5.92 \\
\hline $2.3 \times 2.3 \times 2.3$ & 258 & 8.83 & $2.2 \times 2.2 \times 2.2$ & 275 & 9.00 & $2.2 \times 2.2 \times 1.4$ & 204 & 5.49 \\
\hline $1.9 \times 1.9 \times 1.9$ & 158 & 8.30 & $2.0 \times 2.0 \times 2.0$ & 209 & 8.70 & $1.9 \times 1.9 \times 1.3$ & 140 & 5.20 \\
\hline $1.5 \times 1.5 \times 1.5$ & 76 & 7.55 & $1.8 \times 1.8 \times 1.8$ & 151 & 8.24 & $1.6 \times 1.6 \times 1.0$ & 91 & 4.84 \\
\hline $1.3 \times 1.3 \times 1.3$ & 52 & 6.5 & $1.4 \times 1.4 \times 1.4$ & 66 & 7.33 & $1.0 \times 1.0 \times 0.6$ & 30 & 3.73 \\
\hline \multirow[t]{2}{*}{$1.0 \times 1.0 \times 1.0$} & 22 & 5.2 & $1.2 \times 1.2 \times 1.2$ & 40 & 6.45 & & & \\
\hline & & & $0.9 \times 0.9 \times 0.9$ & 14 & 4.86 & & & \\
\hline \multicolumn{9}{|l|}{ Half-sphere } \\
\hline 3.3 & 476 & 9.6 & 3.3 & 547 & 9.71 & 2.9 & 528 & 6.45 \\
\hline 2.9 & 303 & 9.27 & 2.3 & 307 & 9.33 & 2.7 & 426 & 6.39 \\
\hline 2.7 & 243 & 9.05 & 1.9 & 152 & 8.66 & 2.1 & 190 & 5.85 \\
\hline 2.2 & 138 & 8.41 & 1.4 & 63 & 7.81 & 1.5 & 66 & 5.21 \\
\hline 1.8 & 66 & 7.39 & 1.3 & 43 & 6.8 & 1.2 & 26 & 3.69 \\
\hline 1.3 & 38 & 6.32 & & & & & & \\
\hline \multicolumn{9}{|l|}{ Cylinders ${ }^{b}$} \\
\hline $0.5 \times 9$ & 474 & 8.61 & $0.5 \times 8$ & 546 & 8.87 & $0.5 \times 7$ & 516 & 5.95 \\
\hline $0.5 \times 8$ & 420 & 8.61 & $0.5 \times 7$ & 476 & 8.84 & $0.5 \times 6$ & 441 & 5.95 \\
\hline $0.5 \times 6$ & 315 & 8.6 & $0.5 \times 4$ & 280 & 8.75 & $0.5 \times 5$ & 369 & 5.89 \\
\hline $0.5 \times 4$ & 210 & 8.44 & $0.5 \times 3$ & 210 & 8.67 & $0.5 \times 3.5$ & 264 & 5.82 \\
\hline $0.5 \times 2.7$ & 147 & 8.33 & $0.5 \times 2$ & 140 & 8.5 & $0.5 \times 1.5$ & 117 & 5.47 \\
\hline $0.5 \times 1.5$ & 85 & 8 & $0.5 \times 1.5$ & 98 & 8.29 & $0.5 \times 0.8$ & 63 & 5.08 \\
\hline $0.5 \times 1$ & 54 & 7.41 & $0.6 \times 1.5$ & 147 & 8.69 & $0.5 \times 0.5$ & 42 & 4.57 \\
\hline \multirow[t]{2}{*}{$0.5 \times 0.6$} & 33 & 5.14 & $0.9 \times 1.5$ & 336 & 9.71 & & & \\
\hline & & & $1.1 \times 1.5$ & 497 & 9.81 & & & \\
\hline
\end{tabular}

\begin{tabular}{|c|c|c|c|c|}
\hline \multicolumn{2}{|l|}{ (b) } & \multirow{2}{*}{$\begin{array}{l}\text { Shape/size }(\mathrm{nm}) \\
\text { Icosahedron }\end{array}$} & \multirow{2}{*}{$\begin{array}{l}\text { No. atoms } \\
\text { Decahedron }\end{array}$} & \multirow{2}{*}{ First shell CN } \\
\hline Sphere & Cuboctahedron & & & \\
\hline 3 & 561 & 10.06 & 10.37 & 10.12 \\
\hline 2 & 309 & 9.63 & 10.02 & 9.7 \\
\hline 1.5 & 147 & 8.98 & 9.47 & 9.06 \\
\hline 0.6 & 55 & 7.85 & 8.51 & 7.96 \\
\hline 0.3 & 13 & 5.53 & 6.46 & 5.6 \\
\hline
\end{tabular}

Table 3 Parameters derived from the non-linear least squares fitting of a Hill exponential function (eqn (1)) to the simulated cluster sizes/shapes. The results from the fitting process are shown in the ESI, Fig. $1 \dagger$

\begin{tabular}{|c|c|c|c|c|c|c|c|c|c|c|c|c|}
\hline \multirow[b]{2}{*}{ Shape } & \multicolumn{4}{|l|}{$\mathrm{fcc}$} & \multicolumn{4}{|l|}{ hcp } & \multicolumn{4}{|l|}{ bcc } \\
\hline & $V_{\text {Max }}$ & $k$ & $n$ & $\chi^{2}$ & $V_{\text {Max }}$ & $k$ & $n$ & $\chi^{2}$ & $V_{\text {Max }}$ & $k$ & $n$ & $\chi^{2}$ \\
\hline $\begin{array}{l}\text { Sphere } \\
(\mathrm{NS}=10)\end{array}$ & 12 & $\begin{array}{l}16.74737 \\
( \pm 1.22583)\end{array}$ & $\begin{array}{l}0.48934 \\
( \pm 0.01929)\end{array}$ & 0.0173 & 12 & $\begin{array}{l}14.67494 \\
( \pm 0.72995)\end{array}$ & $\begin{array}{l}0.46135 \\
( \pm 0.01098)\end{array}$ & 0.01014 & 8 & $\begin{array}{l}11.02908 \\
( \pm 0.78355)\end{array}$ & $\begin{array}{l}0.42863 \\
( \pm 0.01237)\end{array}$ & 0.00399 \\
\hline $\begin{array}{l}\Sigma_{\text {All }} \\
(\mathrm{NS}=30-40)^{a}\end{array}$ & 12 & $\begin{array}{l}23.79991 \\
( \pm 2.47564)\end{array}$ & $\begin{array}{l}0.45131 \\
( \pm 0.02673)\end{array}$ & 0.19513 & 12 & $\begin{array}{l}19.0756 \\
( \pm 1.61591)\end{array}$ & $\begin{array}{l}0.43613 \\
( \pm 0.01856)\end{array}$ & 0.1267 & 8 & $\begin{array}{l}23.48406 \\
( \pm 3.24281)\end{array}$ & $\begin{array}{l}0.42183 \\
( \pm 0.03103)\end{array}$ & 0.10529 \\
\hline
\end{tabular}

$\mathrm{NS}=$ number of different shapes/sizes over which the data has been fitted. $V_{\text {Max }}=$ maximum coordination number. ${ }^{a} 30$ particles were profiled for bec structures and 40 for both fcc and hep. 
parameters obtained from an analysis of the foil used in the spectral simulations. In these examples the inclusion of multiple scattering paths did not improve the fit to the data and were ignored (see ESI, Fig. 2†). The influence of crystal shape (cuboctahedra, icosahedra, decahedra, as well as half-spheres, cylinders, cubes and square-based pyramids) on the hypothetical EXAFS of monometallic Pt was then examined. In addition, the influence on the near neighbour distribution on the EXAFS FT data for the two metals in a bimetal cluster was also determined for the 8 model bimetallic structures. Since it can be expected that the Debye-Waller factors for very small particles may strongly deviate from the bulk, we confined our spectral simulations to those for particles $>100$ atoms and $>1 \mathrm{~nm}$ in size.

\section{Results and discussion}

\section{Numerical simulations of monometallic particles}

As mentioned previously, simulations were restricted to cluster sizes of $3 \mathrm{~nm}$ and below. The data are summarised further in Fig. 2, which contains plots of the influence of cluster size/ shape on the first shell coordination number for fcc, hep and bcc structures. For all sets of samples definitive trends can be seen demonstrating a clear influence of particle size and shape for a fixed number of particles on the coordination numberwe note however that these trends are more difficult to differentiate between when considering a typical $\pm 10 \%$ error in the first shell coordination number determination. In most cases, the growth curves begin to plateau on reaching the maximum cluster size sampled. As expected, only small variations in the behavioural trends between the three types of packing can be seen - the main difference being that the maximum first shell coordination number in the bcc structure is only 8 as compared to 12 for fcc and hcp. Dealing only at this stage with the influence of shape on the coordination number, the trend follows that the sphere for a given number of atoms and packing always possesses the highest coordination number and the cylinder typically the lowest. The cause of this difference is the varying extent of under-coordination between the clusters-i.e. the cylinder contains a larger number of atoms arranged at edges and terminal points in the structure than the sphere, with the other structures exhibiting behaviour in between. This effect is demonstrated in Fig. 3 which contains a plot detailing how the trends observed in Fig. 2 show a direct correlation with the different surface to bulk ratios.

The influence of spherical cluster shape on the first shell coordination is small: the decahedron possesses an identical coordination number for the magic clusters to the cuboctahedron, whereas the icosahedrons possess larger coordination numbers for the same cluster size(s), as has been shown previously. ${ }^{8,9}$ The first coordination sphere for the decahedron and the icosahedron should be considered however as split into two shells which for Pt would be 2.75 and $2.89 \AA$ respectively. For both structures the occupancy ratio between the shells is normally split $2: 3$ although here we have simply summed them together since these distances fall within the binning window $(0.15 \AA)$ used in the simulation. Despite the
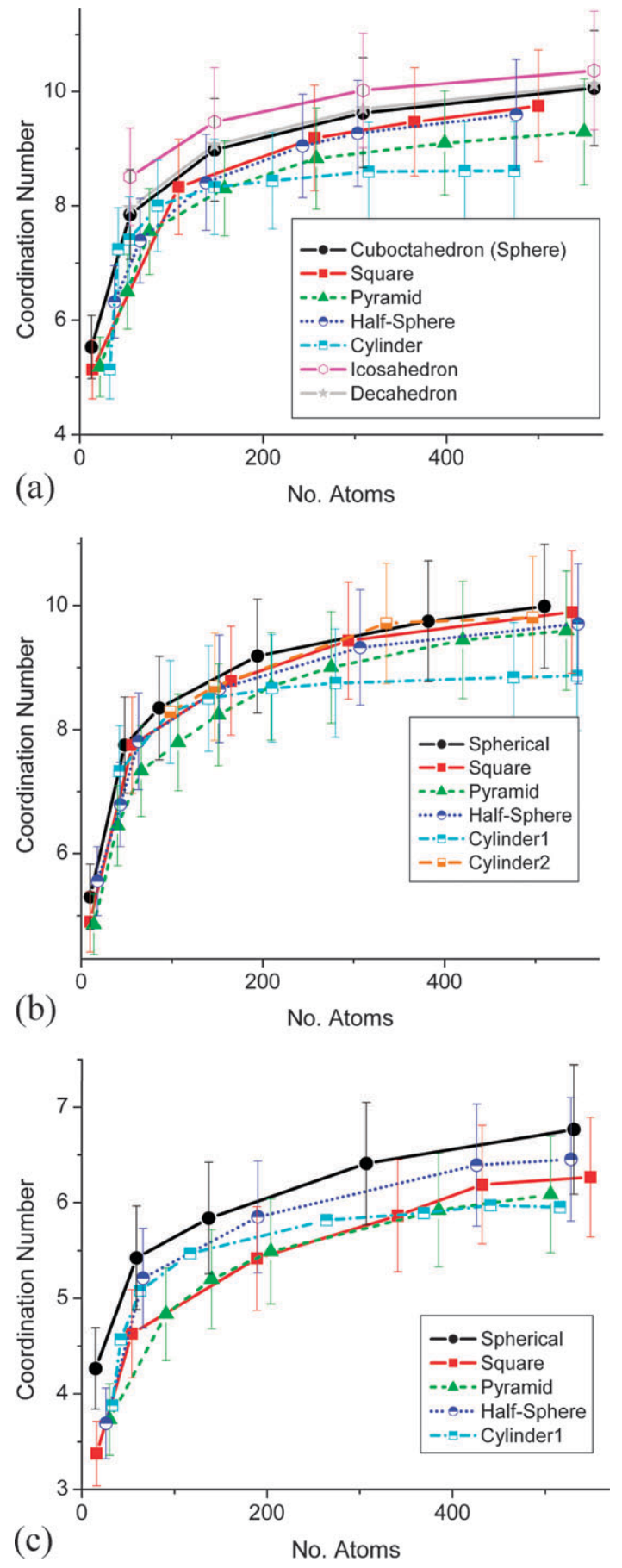

Fig. 2 Influence of the number of atoms in a cluster (size) and its shape on the average first shell coordination sphere for fcc structures (a), hcp structures (b) and bcc structures (c). Note for comparison the icosahedron and decahedron structures are included with the fcc structures. The error bars added indicate a typical $\pm 10 \%$ error in the coordination number determination.

differences in the cluster packing, the surface-to-bulk ratio for the spherical structures remains the same.

For the cylindrical structures a plateauing of both the particle size and surface-to-bulk ratio with the number of atoms in the cluster was observed, equal to 8.6 for $\mathrm{Pt} \mathrm{fcc}$ (316 atoms) and 8.8 for Co hcp (280 atoms) and 5.9 for Fe bcc 
Table 4 EXAFS parameters used to simulate spectra shown in Fig. 7 and 8 and

\begin{tabular}{llllll}
\hline & Shell 1 & Shell 2 & Shell 3 & Shell 4 & Shell 5 \\
\hline Pt-Pt distance & $2.75-3.00$ & $3.90-4.20$ & $4.20-4.90$ & $4.90-5.60$ & $5.60-6.30$ \\
Debye-Waller factor $\left(2 \sigma^{2}\right)$ & 0.01179 & 0.01495 & 0.01771 & 0.02386 \\
Ef & -3.63 & & & \\
S0 & 0.9 & & & \\
\hline
\end{tabular}
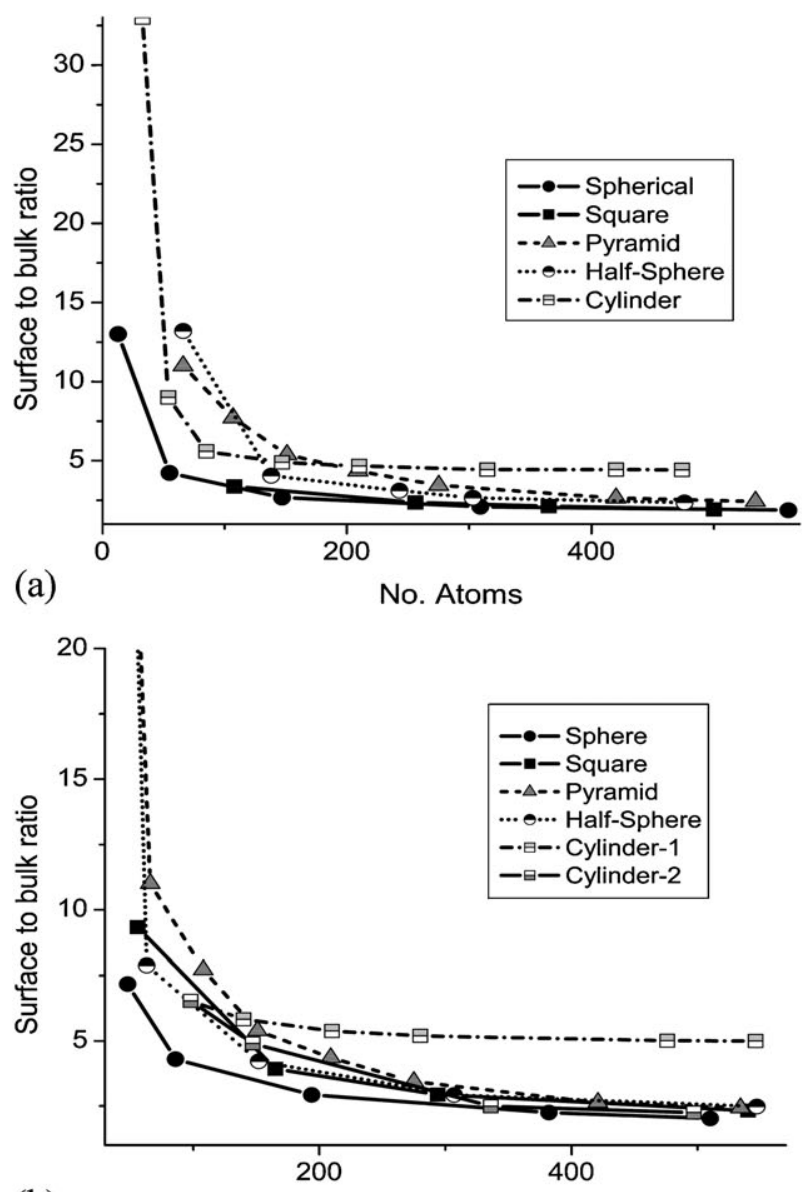

(b)

No. Atoms

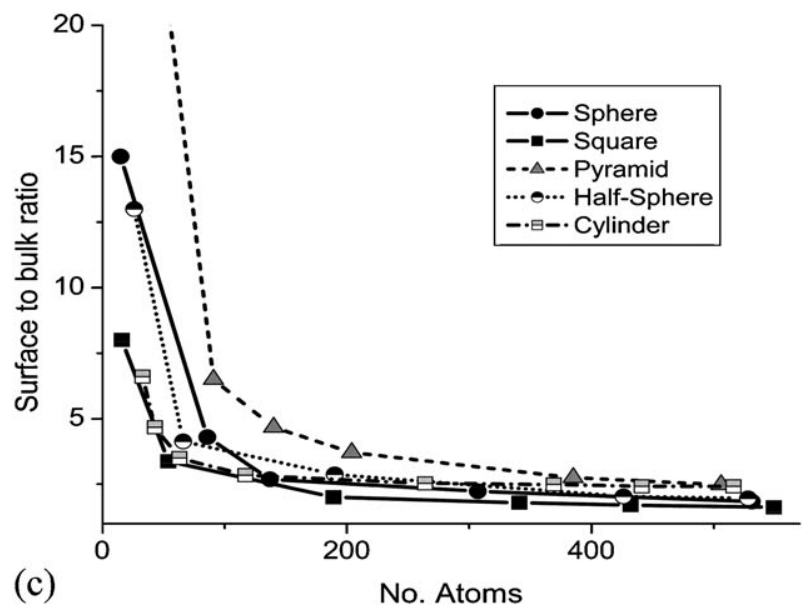

Fig. 3 Surface to bulk ratio plots for fcc (a), hcp (b) and bcc (c) structures.

(370 atoms) - the onset of the plateau in both the Pt fcc and Co hcp structures is probably the same since they possess similar packing efficiency $(74 \%)$ whereas for the $\mathrm{Fe}$ bcc structure the packing efficiency is lower $(68 \%)$ resulting in a much later onset of the plateau. The plateauing phenomenon itself is perhaps not surprising since the cylinders were 'grown' in one dimension (in length at a constant height). Thus it would appear that even though the clusters are bigger there is no accompanying increase in surface-to-bulk ratio and thus the coordination number remains the same.

In order to glean information on the influence of particle shape from coordination numbers determined from a fitting of the EXAFS data, a ratio of the influences of cluster shape on the higher peak intensities has been examined. As mentioned previously, the error in the determination of coordination numbers in EXAFS has been estimated to be around $\pm 10 \%$ for first shell determinations and $\pm 20 \%$ for higher shells; the ratios are therefore subject to error although for the purpose of figure clarity, we do not display error bars in the plot(s).

Fig. 4, 5 and 6 contain plots detailing the influence of particle shape/sizes on the intensity ratios for the fcc, hcp and bec structures respectively. For the fcc structures, 3 ratios are considered, namely the intensity ratio between the first shell and the 2 nd, 3 rd and 4 th shells respectively. The 5 th shell was not included since for the nanoclusters considered in this study, the number of atoms present in this shell is low. Also included in this plot are the data for the icosahedron and decahedron. Since the number of discernible shells for these structures is higher than for the cuboctahedron (due to strain effects), intensity ratios for these structures are plotted as a function of comparable shell position with that of the cuboctahedron rather than the distribution order in which they are found in the RDF. For example, Fig. 4(c) contains a plot of the intensity ratio between the first peak and the fourth peak at $5.50 \AA$ for the fcc structures with peaks at $5.35 \AA$ (icosahedron 6th shell) and $5.45 \AA$ (decahedron 5th shell) respectively. For the bcc structures the last shell (shell 7) and for hcp, shells 3, 6 and 8 were also not included in the variation in intensity ratio again because of their lack of intensity.

As can be seen in Fig. 4, clear differences can be seen between the intensity ratios for the various peaks considered as a function of particle shape/packing. In general and as expected, the growth curves take on an exponential form as they tend towards a theoretically optimum ratio of $0.5,2$ and 1 . A small exception to this behaviour can be seen in the $2: 1$ ratio curve for the half-sphere and cylinder for the intensity ratio below ca. 67 atoms where at first these curves undergo a decrease in intensity ratio reaching an isosbestic point (below 60 atoms) before reverting to the normal expected exponential behaviour. We observe that the most striking differences, illustrating clearly the influence of packing/shape on the coordination numbers on the intensity ratios can be seen in these curves when the clusters are small; typically below 200 atoms 


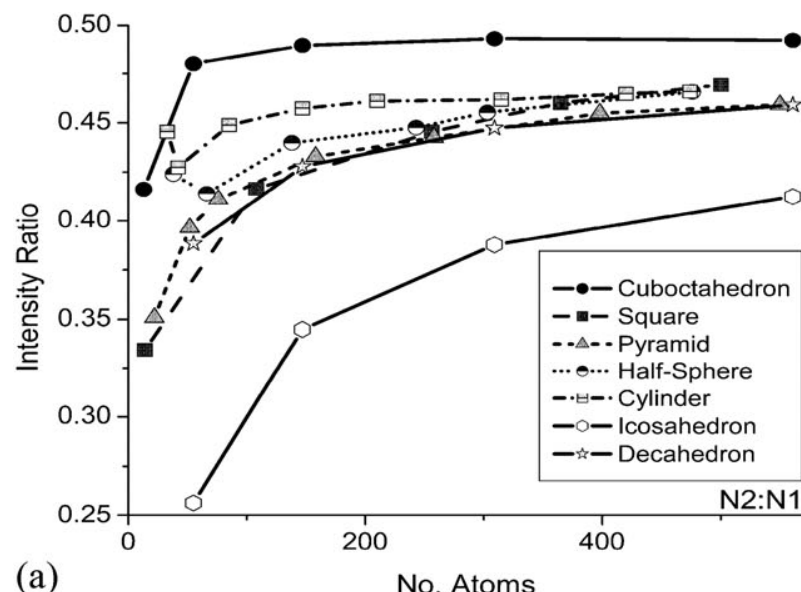

(a)

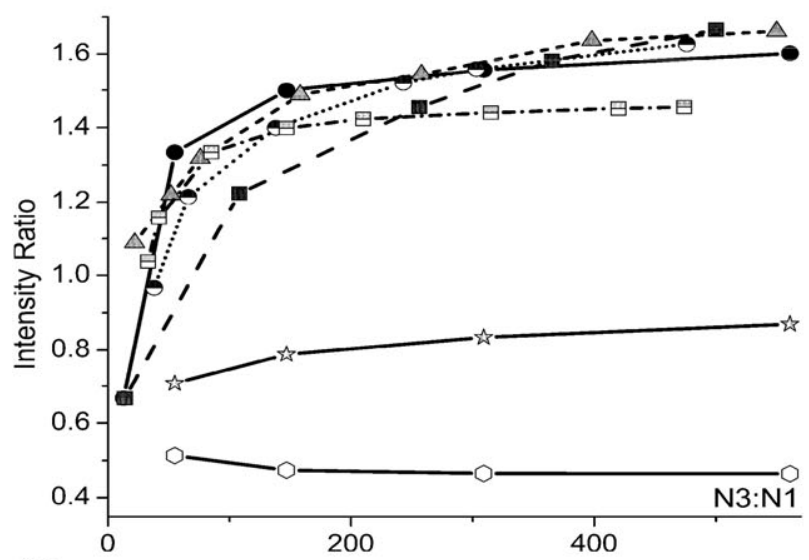

(b)

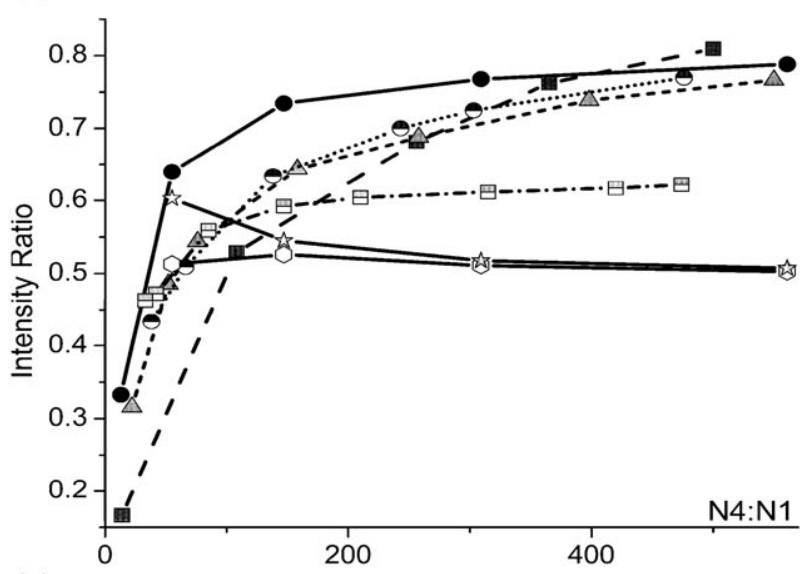

(c)

No. Atoms

Fig. 4 Shell coordination number ratios as a function of atomic packing (fcc, icosahedron and decahedron) and shape for shells $\mathrm{N} 2$ : N1 (a), N3 : N1 (b) and N4:N1 (c).

and more clearly so when they are contain less than 100 atoms. Clearly distinguishable though, irrespective of which shell ratio is considered, are the icosahedron and decahedron. They are characterised by having an often decidedly lower intensity ratio (caused by strain effects splitting the coordination shells) and appear to show much smaller changes in intensity ratio with increasing cluster size. The data presented in Fig. 4 clearly demonstrate that a comparative 3 shell analysis (up to $5.5 \AA$ ) reveals strong variation in the shell intensity ratios as a function of particle shape. ${ }^{10}$

Shown in Fig. 5 are the intensity ratios between the first shell and shells 2, 4, 5 and 7 for Co hcp. We observe that optimum intensity ratios (i.e. for bulk Co hep) for the examined shells should equate to $0.5,1.5,1$ and 1 respectively. As with the fcc structures, with the exception of one or two points on the simulation curves, the growth behaviour can again be described as positive exponential. In contrast to the data presented for the fcc structures, distinguishing between different structures, seems to be more possible when the clusters get larger, particularly for clusters greater than 100 atoms. This is clear to see for shell ratios $\mathrm{N} 2: \mathrm{N} 1$ and $\mathrm{N} 5: \mathrm{N} 1$, where there are strong differences above the 100 atom thresholdalthough we note that such differences may well be more difficult to determine when considering the errors in the coordination number determination.

For the Fe bcc structures, shown in Fig. 6, the intensity ratios between shell 1 and shells 2-6 have been plotted. Here the optimum intensity ratios for the bulk structure should equate to $0.75,1.5,3,1$ and 0.75 respectively. With the exception of the N2:N1 ratio the growth curves show a positive exponential behaviour. In fact the $\mathrm{N} 2: \mathrm{N} 1$ ratio for the majority of the structures studied possess an intensity ratio greater than the theoretical maximum. The cause of this is most likely due to the fact that the two shells actually represent the two atom environments of the bcc lattice. Since truncation of the RDF in the structures occurs by atom rather than entire unit cells the most likely atoms to be removed are those at lattice corners (N1 atoms) rather than those in the centre (N2). This might explain why we observe in the N2:N1 data, deviation/fluctuations in the ratio plots, an apparent strong influence of the shape on shell intensity ratio (particularly for structures with 200 atoms or less) and why the intensity ratios for cube-based structures appears consistently higher than for other structures. However, as with the data presented for hcp structures, it appears that generally it is more difficult to identify structure differences when the clusters are small than when they are large although again we note that such differences may well be more difficult to determine when considering the 10 and $20 \%$ errors in the coordination number determination.

\section{Spectral simulations}

In Fig. 7 is shown both the EXAFS and associated FT for spherical clusters as a function of different packing (cuboctahedron, icosahedron and decahedron respectively) containing 147 atoms. The packing has a clearly dramatic effect on the signal intensities and in fact is negatively correlated with the first shell coordination number (cuboctahedron $\mathrm{N} 1=$ 8.91 , is most intense followed by decahedron $(\mathrm{N} 1=9.06)$ and finally the icosahedron $(\mathrm{N} 1=9.47))$. This is caused by the presence of two near neighbour distances for both the latter two structures (2.75 and 2.82 for the decahedron and 2.75 and $2.89 \AA$ for the icosahedron), leading to a reduced EXAFS amplitude over the entire spectrum caused by destructive interference, whereas the regular packing of the cuboctahedron results in a more coherently scattered signal. ${ }^{5}$ Fig. 8 contains 

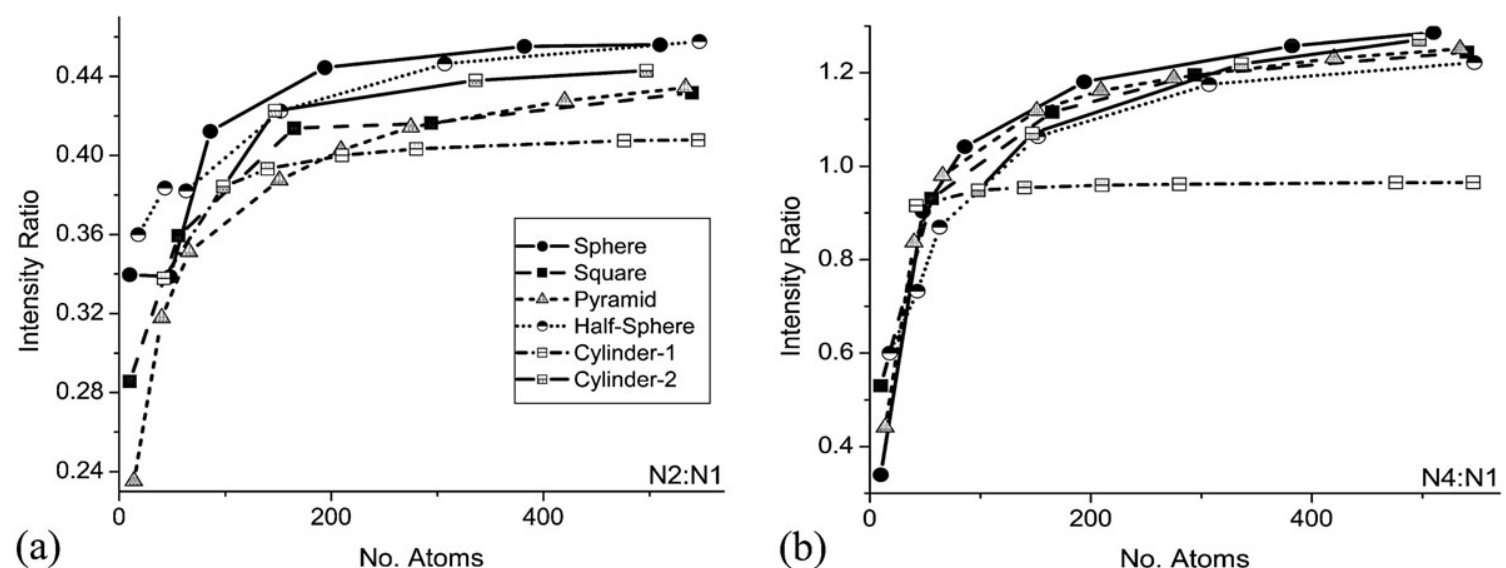

(b)

No. Atoms
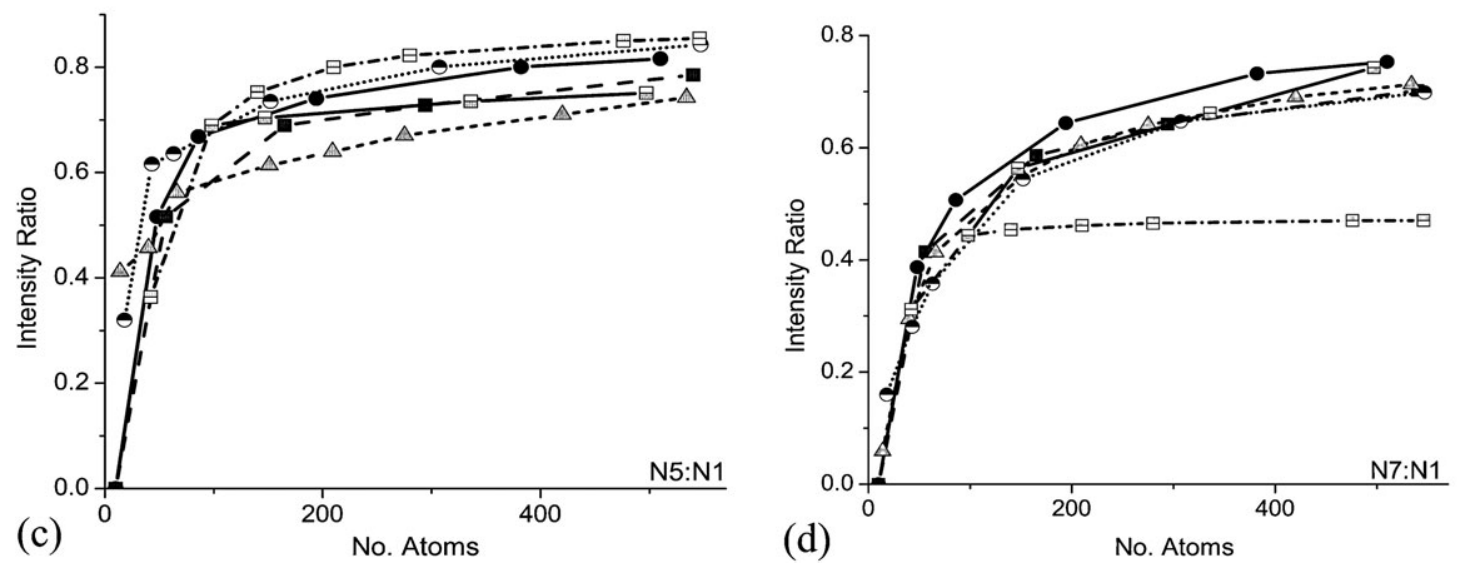

Fig. 5 Shell coordination number ratios for hep as a function of atomic packing and shape for shells N2: N1 (a), N4 : N1 (b), N5 : N1 (c) and N7: N1 (d).

a plot of the influence of the particle shape on the signal intensity, again for structures containing 147 atoms (or in the case for hemi-spherical particles, the intensity was normalised from a cluster containing 138 atoms). Although a clear influence of the shape on the FT peak intensities can be seen - the spherical cuboctahedron possesses the most intense peaks since it has also the highest average coordination number by virtue of having the least number of under coordinated atoms - the differences in the higher peak intensities are noticeable but small.

\section{Bimetallic structures}

The modification of electronic and structural properties of supported metal catalysts is often achieved by the process of alloying. ${ }^{13,37-42}$ However, the degree and process of alloying depends on both the inherent properties of the bimetal species used (i.e. electropositivity and bulk crystal structure) as well as the preparation process and reaction conditions. Such parameters can play an important role in the nature of the resultant bimetal structures (i.e. whether the bimetallic species are distributed randomly or non-randomly (locally clustered)) which in turn will influence the catalytic performance of the bimetallic species. XAFS can be used to interrogate the nature of such bimetal species and in particular can be used to determine both the size of the bimetal species and the distribution of the two metals within the structure. In Fig. 9 and Table 5, we show 6 examples of plausible spherical bimetallic structures $(\mathrm{Pt} / \mathrm{Pd})$ consisting of 137 atoms $(\mathrm{N} 1=$ 8.88) and the determined average first shell coordination numbers calculated for both elements from both perspectives (as if two sets of EXAFS data were collected on the same sample at the two edges). Two other types of bimetallic structure are included in Table 5 which are not depicted in Fig. 9-one entitled 'decorated' and the other 'random $(50 / 50)$ ' where, in contrast to the first random structure, the fractional composition was set to $\mathrm{Pt}=\mathrm{Pd}=0.5$. For some of the samples the fraction $(x)$ of each bimetal varied depending on the nature of the simulated bimetal structure but where possible $\mathrm{Pt} / \mathrm{Pd}$ fractional composition was set so to examine only the influence of the structural distribution on the coordination number.

As can be seen from Table 5, both shape and composition have a large influence on the coordination number from the perspective of both elements. Some general trends can be clearly seen including: 1) that the element representing the largest fraction of the composition possesses the highest overall coordination number and 2) for structures which can be considered as non-random or clustered (core-shell/decorated), large differences in the $\sum N_{\mathrm{PtP}}$ and $\sum N_{\mathrm{PdP}}$ coordination numbers were observed whereas in contrast, for random, non-clustered samples this difference was somewhat smaller. 

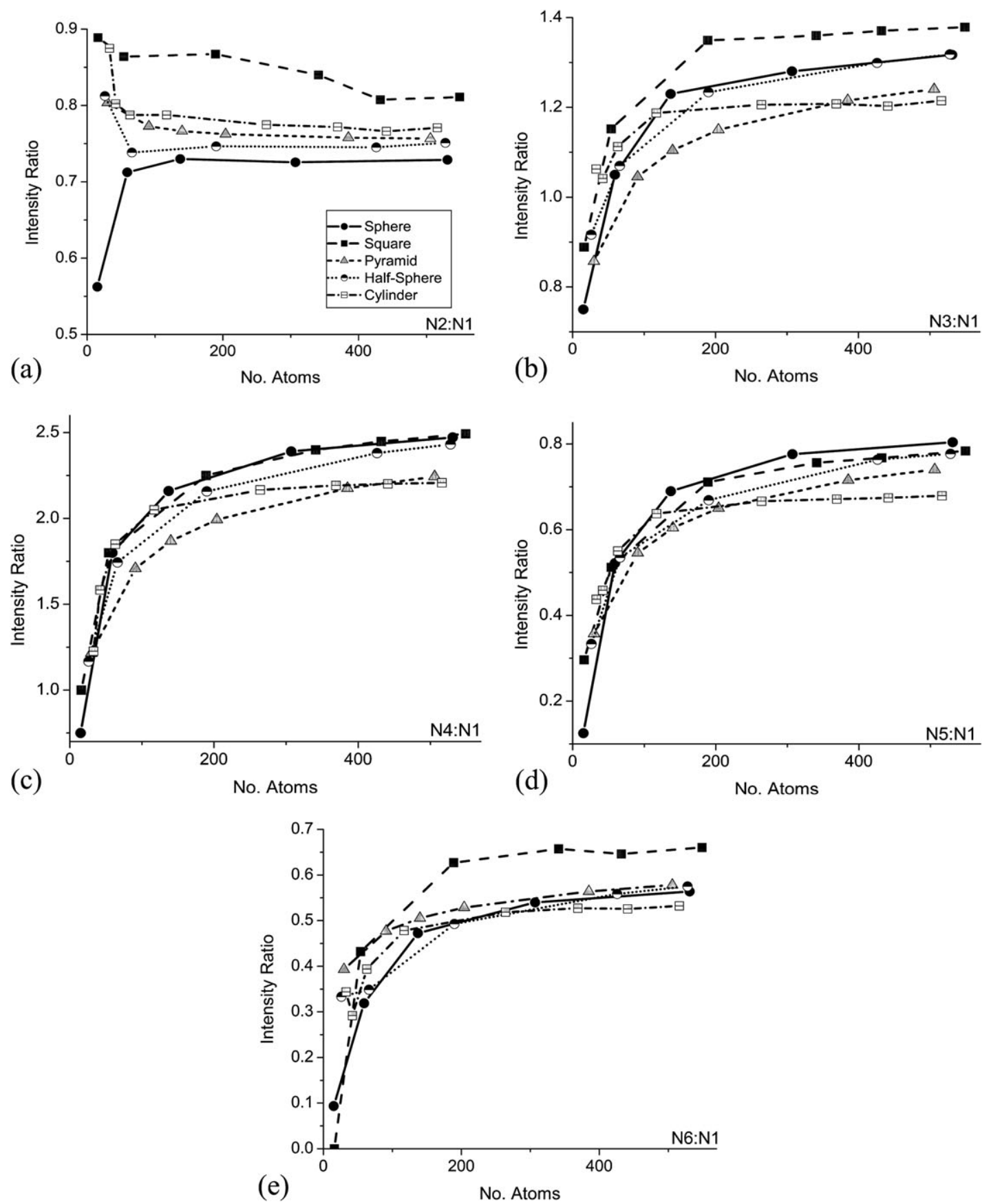

Fig. 6 Shell coordination number ratios for bcc as a function of atomic packing and shape for shells N2 : N1 (a), N3 : N1 (b), N4: N1 (c), N5 : N1 (d) and N6:N1 (e).

We observe at this stage that for random, perfectlymixed alloys, the interrogation of the structure is relatively straightforward. In principle for a 50/50 mixture $(x=0.5)$ consisting of 137 atoms $N_{\mathrm{PtPt}}+N_{\mathrm{PtPd}}=N_{\mathrm{PtP}}$ (single component particle) $=8.88 .{ }^{11}$ When the amount of each element present is not unity it is also necessary to take into account the fractional composition of the elements present in order to obtain this information using the following expression:

$$
N_{\mathrm{PdP}}=x_{\mathrm{Pt}} n_{\mathrm{Pt}} P+x_{\mathrm{Pd}} n_{\mathrm{PdP}}
$$

The equation requires that EXAFS information from both element perspectives be available since the terms $n_{\mathrm{PtM}}$ and $n_{\mathrm{PdM}}$ represent the total first shell coordination number (i.e. both components of a PtPd system). This was previously shown by Frenkel to work for random alloys but as we demonstrate here, is also valid for highly clustered samples. ${ }^{11}$ For example for the core-shell structure, $N_{\mathrm{PdP}}$ evaluates to 8.87 .

As a means of measuring the degree of local clustering within the models on the bimetallic structures we have also calculated a short-range order parameter first introduced 

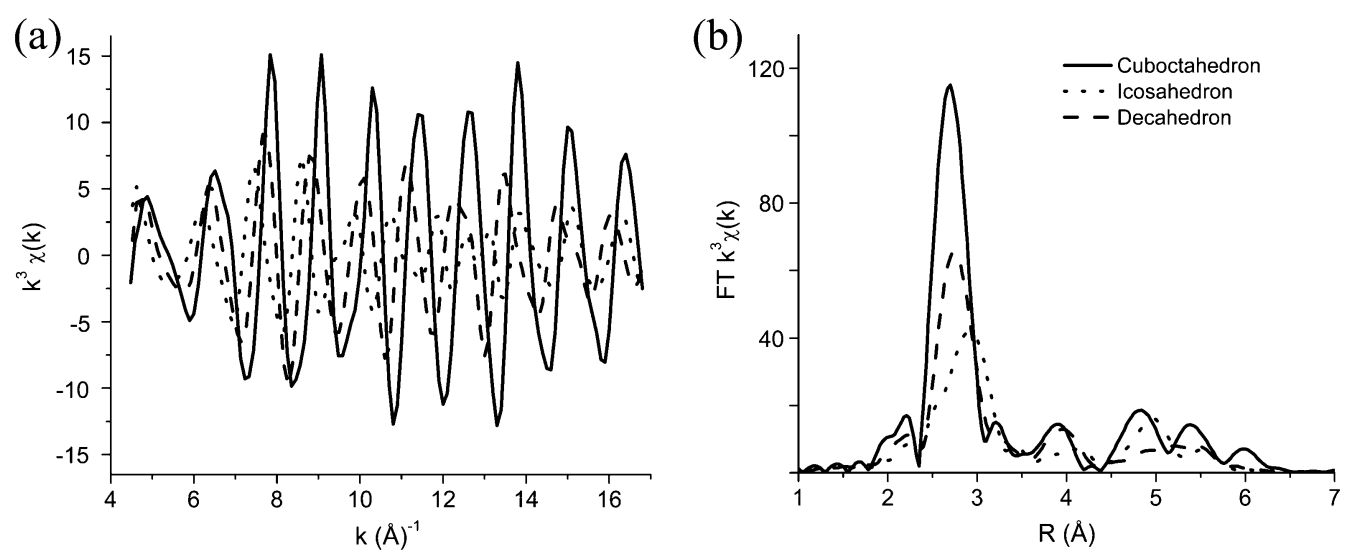

Fig. 7 Simulated EXAFS data (a) and associated FT (b) for 147 atom Pt-containing cuboctahedron, icosahedron and decahedron. The simulations were performed using the parameters listed in Table 3. Note that the maximum in the intensity of the signal for the first neighbour scattering contribution (Pt-Pt) is shifted by $c a .0 .04 \AA$ (decahedron) and by $c a .0 .21 \AA$ (icosahedron), respectively, as a consequence of the splitting of the first coordination sphere. This splitting is responsible for the presence of a shoulder at $2.60 \AA$ in the icosahedron.

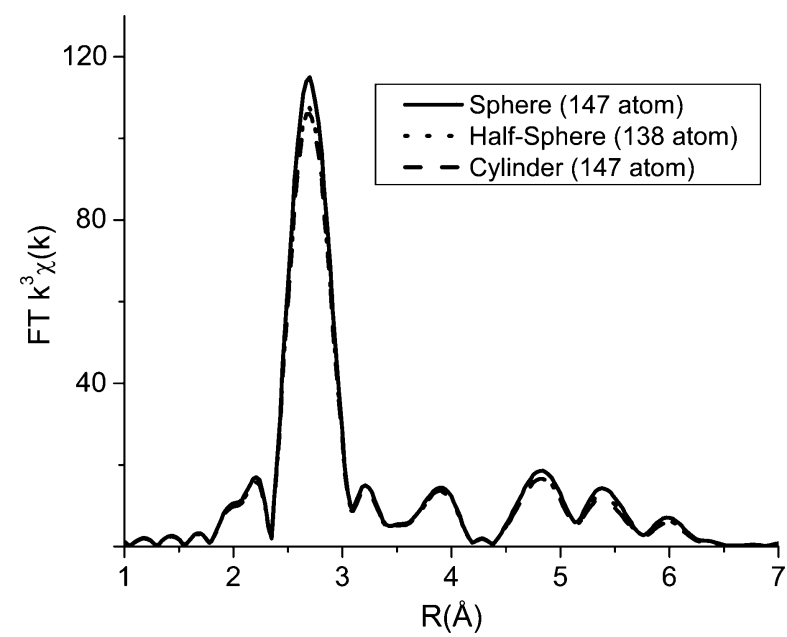

Fig. 8 Influence of cluster shape on the simulated EXAFS FT data. The data have been taken from a combination of that which is shown in Table 2 and Fig. 4.

by Cowley. ${ }^{43}$ The expression summarises the degree of segregation as a function of the ratio $N_{\mathrm{PtPd}} / N_{\mathrm{PtPt}}+N_{\mathrm{PtPd}}$. This is expressed more clearly in eqn (3).

$$
\alpha=\frac{1-N_{\mathrm{PtPd}} / N_{\sum \mathrm{PtPt}}+\mathrm{PtPd}}{X_{\mathrm{Pd}}}
$$

As can be seen from Table 5, a value close to zero is consistent with the formation of a well-dispersed random alloy, whereas negative (or positive if calculated with respect to $x_{\mathrm{Pt}}$ ) values are consistent with the tendency towards clustering. We consider here the possibility, from an empirical analysis of the data in Table 5, whether any trends can be seen in the data for these bimetallic samples and whether this simple analysis can be used to infer structural information/bimetal distribution on unknown bimetallic samples. Since the local environment for each element can differ markedly in each sample, the simplest method should try to evaluate $\alpha$ as a whole. As can be seen in Table 5, we have examined two methods to do this: a difference method $\left(\alpha_{\mathrm{Pt}}-\alpha_{\mathrm{Pd}}=\Delta_{\mathrm{PtPd}}\right)$ and a summation method $\left(\alpha_{\mathrm{Pt}}+\alpha_{\mathrm{Pd}}=\sum_{\alpha}\right)$. In general we can conclude that somewhat expectedly, the difference method seems more sensitive to determining the similarities between the bimetal environments whereas the summation method is sensitive to the extent of bimetal interaction. Clearly, therefore, both parameters need to be evaluated in order to infer the characteristics of a bimetallic sample since one analysis method alone is insufficient. For example, the difference method is sensitive to systems/structures in which the two bimetal sites are identical (bi-cluster structure $\Delta=0$, random $\Delta=0.08$ ) but not to those in which the bimetal sites are entirely different (bi-cluster inhomo $\Delta=-0.02$ ). When used in conjunction with the summation method however, the differences in these structures become more apparent, returning values of $\sum=-1.96$ (bi-cluster), -0.08 (random) and -1.62 (bi-cluster inhomo) respectively. The summative method also seems to be more sensitive to the extent of randomness within the samples. Thus samples in which values of $\sum_{\alpha}$ are highly negative (tending towards -2) can be described as highly non-random (clustered), whereas values tending towards 0 are highly random.

Although the structure of a bimetallic species is difficult to unequivocally determine, the exact composition is more straightforward. This can be achieved from an analysis of the coordination numbers from the second element perspective obtained for both elements. Thus from eqn (3).

$$
\begin{aligned}
& N_{\mathrm{PtPd}} / N_{\mathrm{PdPt}} \sim x_{\mathrm{Pd}}: x_{\mathrm{Pt}} \sim \gamma \\
& \frac{1}{1+\gamma}=x_{\mathrm{Pt}}
\end{aligned}
$$

Thus in eqn (3) we introduce a new term $(\gamma)$ which represents a ratio of the elements in the bimetal cluster. This can for example be clearly seen in Table 4 whereby for the core-shell structure $N_{\mathrm{PtPd}} / N_{\mathrm{PdPt}}=3.86 / 2.92=\gamma=1.32$ and $1 / 2.32=0.43$ accordingly.

\section{Spectral simulations of bimetallic species}

The simulated spectra for the bimetallic structures, utilising data obtained from the respective $\mathrm{Pt}$ and $\mathrm{Pd}$ foils, are shown in 
(a)

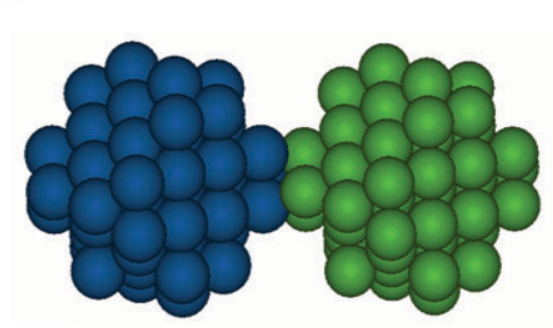

(c)

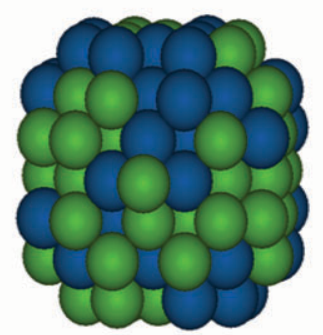

(e)

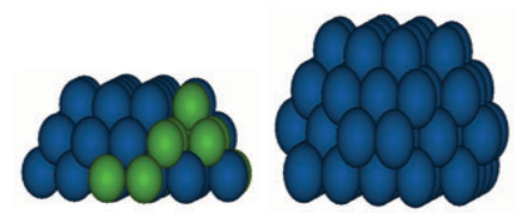

(b)

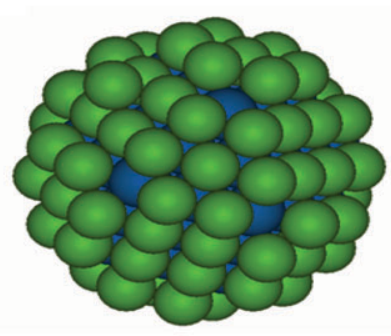

(d)

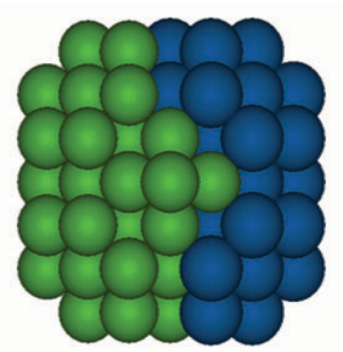

(f)

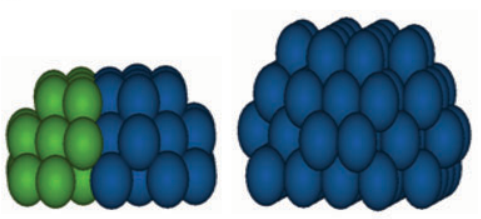

Fig. 9 Hypothetical bimetallic clusters $(\mathrm{Pt}=$ blue, $\mathrm{Pd}=$ green): (a) bi-cluster; (b) core-shell; (c) random; (d) single-half; (e) bi-cluster homo; (f) bi-cluster inhomo.

Table 5 1st shell coordination numbers for PtPd bimetallic systems

\begin{tabular}{llllllllllrrr}
\hline Cluster Type & $\mathrm{Pt}-\mathrm{Pt}$ & $\mathrm{Pt}-\mathrm{Pd}$ & $\sum N_{\mathrm{PtP}}$ & $\mathrm{Pd}-\mathrm{Pd}$ & $\mathrm{Pd}-\mathrm{Pt}$ & $\sum N_{\mathrm{PdP}}$ & $x_{\mathrm{Pt}}$ & $x_{\mathrm{Pd}}$ & $\alpha_{\mathrm{Pt}}$ & $\alpha_{\mathrm{Pd}}$ & $\Delta \alpha$ & $\sum \alpha$ \\
\hline Bi-cluster & 8.73 & 0.07 & 8.80 & 8.73 & 0.07 & 8.80 & 0.5 & 0.5 & -0.98 & -0.98 & 0 \\
Single-Half & 7.08 & 1.58 & 8.66 & 7.85 & 1.19 & 9.04 & 0.43 & 0.57 & -0.68 & -0.69 & -1.96 \\
Core-shell & 7.73 & 3.86 & 11.59 & 3.90 & 2.92 & 6.82 & 0.43 & 0.57 & -0.42 & 0 & -0.42 & -1.37 \\
Random & 3.63 & 4.80 & 8.42 & 5.59 & 3.63 & 9.22 & 0.43 & 0.57 & 0 & -0.08 & 0.08 & -0.08 \\
Decorated & 3.43 & 3.00 & 6.43 & 8.73 & 0.77 & 9.50 & 0.21 & 0.78 & -0.18 & -0.9 & 0.72 & -1.08 \\
Random (50/50) & 5.41 & 4.26 & 9.68 & 4.20 & 3.88 & 8.09 & 0.5 & 0.5 & -0.12 & -0.08 & -0.04 & -0.2 \\
Bi-cluster homo & 7.63 & 0.92 & 8.55 & 3.25 & 4.33 & 7.58 & 0.82 & 0.18 & -0.39 & -0.31 & -0.08 & -0.7 \\
Bi-cluster inhomo & 7.63 & 0.24 & 7.88 & 5.92 & 1.17 & 7.08 & 0.82 & 0.18 & -0.82 & -0.8 & -0.02 & -1.62 \\
\hline
\end{tabular}

Fig. 10. For simplification, only the first shell(s) contributions are simulated. Since the unit cell of $\mathrm{Pd}$ is similar to that of $\mathrm{Pt}$ (3.89 and $3.92 \AA)$, the bond distances $\mathrm{Pt}-\mathrm{Pt}(\mathrm{Pd}-\mathrm{Pd})$ and $\mathrm{Pt}-\mathrm{Pd}(\mathrm{Pd}-\mathrm{Pt})$ were set to 2.75 and $2.73 \AA$ respectively. ${ }^{37}$ Interestingly, two main contributions can be seen in the phase-corrected $\mathrm{Pt} \mathrm{L}_{\mathrm{III}}$-edge FT spectra at $c a .2 .73 \AA$ and $2.46 \AA$, respectively. In contrast for the Pd K-edge FT spectra a strong peak is observed at $c a$. $2.69 \AA$ with a shoulder at $2.25 \AA$. As can be seen from a comparison of the spectra shown in Fig. 10 and with the data presented in Table 5, in both spectra, the intensity of the peaks at high $r$ is related to the degree of monometallic bond formation (i.e. $\mathrm{Pt}-\mathrm{Pt}$ bonds in (a) and $\mathrm{Pd}-\mathrm{Pd}$ in (b)). Conversely, this trend is reversed for the peak intensity at low $r$ and reflects an increasing degree of bimetallic bond formation and therefore an increasing extent of random alloy formation. The low $r$ peaks are too short to correspond to actual chemical bond distances and are caused by a 'pi phase flip' in the backscattering amplitude from $6 \AA$ for elements where $Z>78$; a phenomenon described as the generalised Ramsauer-Townsend effect (GRT). ${ }^{44}$ Increasing the number of bimetallic bonds therefore exacerbates this splitting/the intensity of the low $r$ peak - this is much less pronounced in the Pd K-edge FT data since for the simulations shown here, there are typically fewer Pt atoms than Pd. This has however been observed previously in the Pd K-edge FT data and also appears more clear in the simulations performed with hypothetical intensity ratios as shown in the supplementary information (ESI, Fig. $3 \dagger$ ). As demonstrated more clearly in this Figure, increasing amounts of Pt causes variation in the intensities and positions of both peaks in both Pd K-edge and Pt $\mathrm{L}_{\mathrm{III}}$-edge FT spectra. We observe however, (ESI, Fig. 3(c) and $(\mathrm{d}) \dagger)$ that such peak splitting/peak evolution is also 

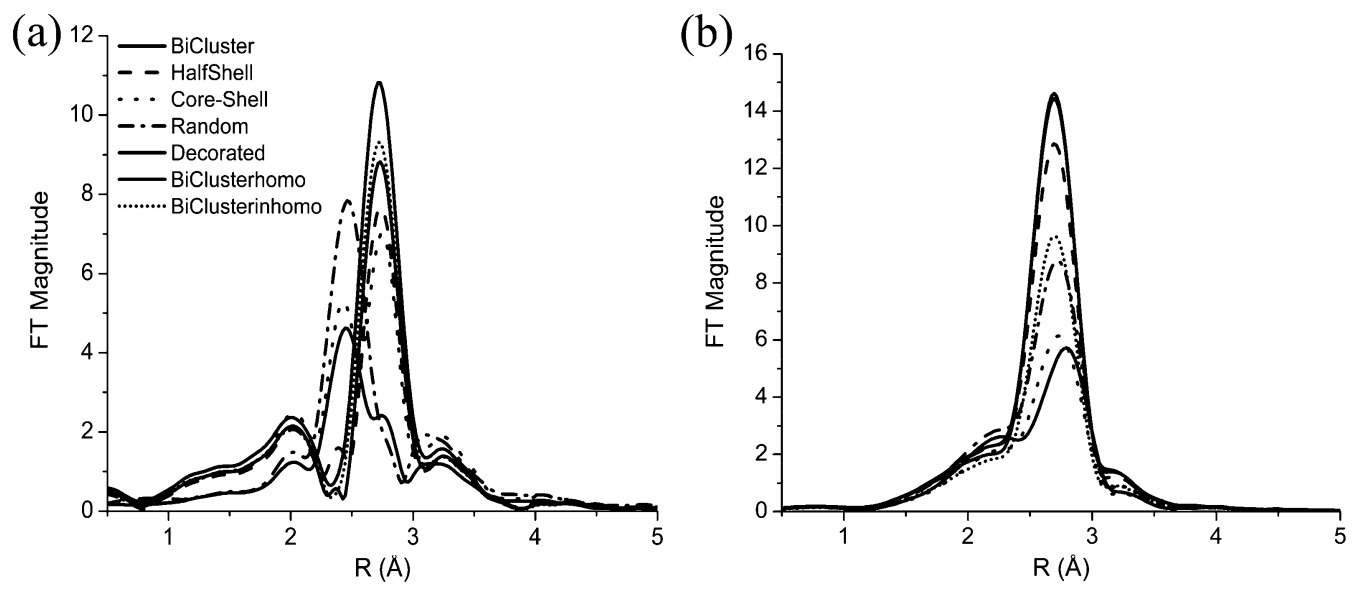

Fig. 10 Influence of bimetal structure on the EXAFS FT data at the Pt edge (a) and Pd edge (b).

observed by increasing $\Delta r$ (particularly $>0.1 \AA$ ) between the 2 metals, although this time resulting in the formation of new peaks at high $r$ in both FT spectra. In the Pd K-edge FT spectra the high $r$ peak appears in the position corresponding to the Pd shell in the Pt $\mathrm{L}_{\mathrm{III}^{-}}$-edge FT spectra but at slightly higher values for the Pt shell in the Pd K-edge FT spectra. We observe therefore that both intensity (influenced by both the coordination number and Debye-Waller factor) and $\Delta r$ can result in a splitting of the FT main peak intensity at both edges, although differences in the coordination number tend to lead to the presence of low $r$ peak formation whereas differences in $\Delta r$ leads to appearances of high $r$ peak formation.

\section{Conclusions}

The influence of atom packing and shape for fcc, hcp and bcc monometallic structures and the bimetal distribution for spherical bimetallic structures, on the coordination number(s) and resultant simulated EXAFS spectra have been examined. For the monometallic structures, a comparative shell intensity ratio analysis, as proposed previously by Jentys for fcc structures, ${ }^{10}$ was also performed in order to examine the influence of shape on the coordination number. We observe that clear differences in the shell occupancy intensity ratios can be seen as a function of size and shape for all types of crystal packing examined. The influence of the shape on the coordination shells is manifested as a function of the surface-to-bulk aspect(s) caused by a variation in the aspect ratios of the shapes in question. From the data however, for each type of packing analysed there appears to exist a size 'regime' over which it is more possible to discriminate between different types of structure accordingly. For fcc and hcp packing, structures comprising less than 200 atoms and particularly less than 100 atoms, seem to exhibit strong variation in coordination shell number as a function of shape. For bcc structures this dependency of the coordination shell number for small clusters is much less pronounced and in some cases (for higher shell ( $>\mathrm{N} 4$ ) coordination numbers) even exhibits the opposite trend in that the difference in coordination number as a function of shape becomes more marked with an increasing number of atoms. The simulations of the data suggest however that the differences in the actual spectra are small thus making a distinction between shapes on EXAFS data alone becomes more difficult as the samples become more polydisperse. Trends observed during dynamic measurement (e.g. particle elongation, sintering, surface reaction/flattening, bimetal particle restructuring etc... during a catalytic reaction) ${ }^{45-47}$ for which EXAFS has been often applied, could be deduced from such a comparison of coordination shells. However, we note that in the light of the developments of nanoscopic X-ray based techniques, ${ }^{48,49}$ data analysis and extraction methodology (i.e. PCA analysis/differential spectrum) as well as improvements in the controlled synthesis of nanoparticles allowing for the development of 'designer catalysts', it may well be that sample polydispersity will become less of a problem.

\section{Acknowledgements}

A. M. B. and B. M. W. would like to thank NWO for a VENI and VICI award respectively. In addition the CCLRC (Daresbury Laboratory) and NWO (DUBBLE@ESRF) is thanked for access to synchrotron radiation facilities.

\section{References}

1 A. T. Bell, Science, 2003, 299, 1688-1691.

2 K. P. de Jong, Curr. Opin. Solid State Mater. Sci., 1999, 4, 55-62.

3 V. Petkov, Mater. Today, 2008, 11, 28-38.

4 J. D. Grunwaldt and A. Baiker, Phys. Chem. Chem. Phys., 2005, 7, 3526-3539.

5 Y. Iwasawa, J. Catal., 2003, 216, 165-177.

6 M. A. Newton, A. J. Dent and J. Evans, Chem. Soc. Rev., 2002, 31, 83-95.

7 R. B. Greegor and F. W. Lytle, J. Catal., 1980, 63, 476-486.

8 R. E. Benfield, J. Chem. Soc., Faraday Trans., 1992, 88, 1107-1110.

9 J. M. Montejano-Carrizales, F. Aguilera-Granja and J. L. Moran-Lopez, Nanostruct. Mater., 1997, 8, 269-287.

10 A. Jentys, Phys. Chem. Chem. Phys., 1999, 1, 4059-4063.

11 A. I. Frenkel, Z. Kristallogr., 2007, 222, 605-611.

12 E. Sacaliuc, A. M. Beale, B. M. Weckhuysen and T. A. Nijhuis, J. Catal., 2007, 248, 235-248.

13 G. A. Somorjai and J. Y. Park, Top. Catal., 2008, 49, 126-135.

14 J. L. Wang, J. Lian, J. R. Greer, W. D. Nix and K. S. Kim, Acta Mater., 2006, 54, 3973-3982.

15 J. L. Burt, J. L. Elechiguerra, J. Reyes-Gasga, J. M. MontejanoCarrizales and M. Jose-Yacaman, J. Cryst. Growth, 2005, 285, 681-691. 
16 J. Reyes-Gasga, S. Tehuacanero-Nunez, J. M. MontejanoCarrizales, X. X. Gao and M. Jose-Yacaman, Top. Catal., 2007, 46, 23-30.

17 C. L. Johnson, E. Snoeck, M. Ezcurdia, B. Rodriguez-Gonzalez, I. Pastoriza-Santos, L. M. Liz-Marzan and M. J. Hytch, Nat. Mater., 2008, 7, 120-124.

18 F. Tielens, J. Andres, M. Van Brussel, C. Buess-Hermann and P. Geerlings, J. Phys. Chem. B, 2005, 109, 7624-7630.

19 J. R. Renzas, Y. Zhang, W. Huang and G. A. Somorjai, Catal. Lett., 2009, 132, 317-322.

20 K. Pirkkalainen and R. Serimaa, J. Appl. Crystallogr., 2009, 42, 442-447.

21 G. L. Bezemer, J. H. Bitter, H. Kuipers, H. Oosterbeek, J. E. Holewijn, X. D. Xu, F. Kapteijn, A. J. van Dillen and K. P. de Jong, J. Am. Chem. Soc., 2006, 128, 3956-3964.

22 O. Borg, P. D. C. Dietzel, A. I. Spjelkavik, E. Z. Tveten, J. C. Walmsley, S. Diplas, S. Eri, A. Holmen and E. Ryttera, J. Catal., 2008, 259, 161-164.

23 M. S. Chen and D. W. Goodman, Catal. Today, 2006, 111, 22-33.

24 J. P. den Breejen, P. B. Radstake, G. L. Bezemer, J. H. Bitter, V. Froseth, A. Holmen and K. P. de Jong, J. Am. Chem. Soc., 2009, 131, 7197-7203.

25 K. Koga and K. Sugawara, Surf. Sci., 2003, 529, 23-35.

26 A. M. Saib, A. Borgna, J. V. de Loosdrecht, P. J. van Berge and J. W. Niemantsverdriet, Appl. Catal., A, 2006, 312, 12-19.

27 E. de Smit, A. M. Beale, S. Nikitenko and B. M. Weckhuysen, J. Catal., 2009, 262, 244-256.

28 E. de Smit, I. Swart, J. F. Creemer, C. Karunakaran, D. Bertwistle, H. W. Zandbergen, F. M. F. de Groot and B. M. Weckhuysen, Angew. Chem., Int. Ed., 2009, 48, 3632-3636.

29 F. Morales, D. Grandjean, A. Mens, F. M. F. de Groot and B. M. Weckhuysen, J. Phys. Chem. B, 2006, 110, 8626-8639.

30 B. Mierzwa, J. Alloys Compd., 2004, 362, 178.

31 L. J. E. Hofer and W. C. Peebles, J. Am. Chem. Soc., 1947, 69, 893-899.

32 J. Haglund, A. F. Guillermet, G. Grimvall and M. Korling, Phys. Rev. B: Condens. Matter, 1993, 48, 11685-11691.

33 D. R. Wilburn and W. A. Bassett, Am. Miner., 1978, 63, 591-596.
34 G. W. Turner, R. L. Johnston and N. T. Wilson, J. Chem. Phys., 2000, 112, 4773-4778.

35 C. Antoniak, M. Spasova, A. Trunova, K. Fauth, F. Wilhelm, A. Rogalev, J. Minar, H. Ebert, M. Farle and H. Wende, J. Phys.: Condens. Matter, 2009, 21.

36 S. Nikitenko, A. M. Beale, A. M. J. van der Eerden, S. D. M. Jacques, O. Leynaud, M. G. O'Brien, D. Detollenaere, R. Kaptein, B. M. Weckhuysen and W. Bras, J. Synchrotron Radiat., 2008, 15, 632-640.

37 R. Roldan, A. M. Beale, M. Sanchez-Sanchez, F. J. RomeroSalguero, C. Jimenez-Sanchidrian, J. P. Gomez and G. Sankar, J. Catal., 2008, 254, 12-26.

38 A. J. Dent, J. Evans, S. G. Fiddy, B. Jyoti, M. A. Newton and M. Tromp, Faraday Discuss., 2008, 138, 287-300.

39 D. I. Garcia-Gutierrez, C. E. Gutierrez-Wing, L. Giovanetti, J. M. Ramallo-Lopez, F. G. Requejo and M. Jose-Yacaman, J. Phys. Chem. B, 2005, 109, 3813-3821.

40 J. M. Thomas, R. Raja, B. F. G. Johnson, S. Hermans, M. D. Jones and T. Khimyak, Ind. Eng. Chem. Res., 2003, 42, $1563-1570$.

41 M. Harada, K. Asakura and N. Toshima, J. Phys. Chem., 1993, 97, 5103-5114.

42 M. R. Knecht, M. G. Weir, A. I. Frenkel and R. M. Crooks, Chem. Mater., 2008, 20, 1019-1028.

43 J. M. Cowley, Phys. Rev., 1965, 138, A1384-A1389.

44 A. G. McKale, B. W. Veale, A. P. Paulikas, S. K. Chan and K. G. S, Phys. Rev. B: Condens. Matter, 1988, 38, 10919.

45 A. M. Molenbroek, S. Helveg, H. Topsoe and B. S. Clausen, Top. Catal., 2009, 52, 1303-1311.

46 M. A. Newton, Chem. Soc. Rev., 2008, 37, 2644-2657.

47 R. Zanella, S. Giorgio, C. H. Shin, C. R. Henry and C. Louis, J. Catal., 2004, 222, 357-367.

48 A. Erko and I. Zizak, Spectrochim. Acta, Part B, 2009, 64, 833-848.

49 E. de Smit, I. Swart, J. F. Creemer, G. H. Hoveling, M. K. Gilles, T. Tyliszczak, P. J. Kooyman, H. W. Zandbergen, C. Morin, B. M. Weckhuysen and F. M. F. de Groot, Nature, 2008, 456, 222-U239. 